\title{
Potential Efficacy of Multiple-shot Long-pulsed 1,064-nm Nd:YAG in Nonablative Skin Rejuvenation: A Pilot Study
}

\author{
Young-Koo Kim \\ Hae-Jin Lee ${ }^{2}$ \\ Jihee $\mathrm{Kim}^{3}$
}

\footnotetext{
${ }^{1}$ Yonsei Star Skin and Laser Clinic, Seoul, Korea

${ }^{2}$ Yonsei A\&B Skin Clinic, Seoul, Korea

${ }^{3}$ Yongin Severance Hospital, Yonsei University

College of Medicine, Yongin, Korea
}

Received December 21, 2020

Accepted December 21, 2020

\author{
Correspondence \\ Jihee Kim \\ Department of Dermatology, Yongin Severance \\ Hospital, Yonsei University College of Medicine, \\ 363 Dongbaekjukjeondaero, Giheung-gu, Yongin \\ 16995, Korea \\ Tel.: +82-31-5189-8891 \\ Fax: +82-31-5189-8567 \\ E-mail: mygirljiheedyuhs.ac
}

(C) Korean Society for Laser Medicine and Surgery

(c) This is an open access article distributed under the terms of the Creative Commons Attribution NonCommercial License (http://creativecommons.org/ licenses/by-nc/4.0) which permits unrestricted noncommercial use, distribution, and reproduction in any medium, provided the original work is properly cited.

\section{Background and Objectives}

The ultimate goal in current skin rejuvenation practice is to achieve a good result with minimal pain and downtime. Nonablative skin rejuvenation (NSR) is one technique. The efficacy of the long-pulsed 1064 $\mathrm{nm}$ Nd:YAG laser (LPNDY) has not been assessed in NSR.

\section{Materials and Methods}

Three target areas were selected (bilateral cheeks and glabellar region) in six volunteer subjects. A LPNDY with an integral skin temperature monitor delivered three stacked shots to each target area (1064 nm, 12 $\mathrm{mm}$ spot, $13 \mathrm{~J} / \mathrm{cm}^{2}, 1 \mathrm{~Hz}$ ) without any skin cooling or anesthesia. The skin temperature was recorded before, during, and after each set of shots using the system monitor and in real-time using a high-sensitivity $\left( \pm 0.001^{\circ} \mathrm{C}\right)$ near-infrared video camera. The skin reaction was observed with the naked eye, and pain and discomfort were assessed by the subjects during and after treatment.

\section{Results}

The subjects reported a mild feeling of heat with no discomfort during or after the test treatments. Mild erythema was observed around the treatment areas, without noticeable edema. A series of three ascending skin temperature stepwise peaks, with a decrease in skin temperature towards the baseline after the third shot, was observed consistently. The mean temperatures for shots 1,2 , and 3 for the cheeks were $39.5^{\circ} \mathrm{C}$, $42.0^{\circ} \mathrm{C}$, and $44.4^{\circ} \mathrm{C}$, respectively, and for the glabella, $40.8^{\circ} \mathrm{C}, 43.9^{\circ} \mathrm{C}$, and $46.2^{\circ} \mathrm{C}$, respectively. Similar ranges were indicated on the system integral temperature monitor.

\section{Conclusion}

A set of three stacked pulses with the LPNDY at a low fluence achieved ideal dermal temperatures to achieve some dermal remodeling but without any downtime or adverse events. The temperature data from the integral thermal sensor matched the video camera measurements with practical accuracy for skin rejuvenation requirements. These data suggest that LPNDY would satisfy the necessary criteria to achieve effective NSR, but further studies will be needed to assess the actual results in clinical practice.

\section{Key words}

Nonablative skin rejuvenation; Long-pulsed Nd:YAG laser; Shot stacking; Heat shock proteins, Integral IR thermal sensor 


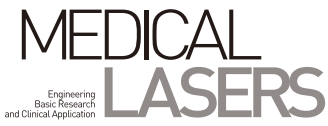

\section{INTRODUCTION}

The rejuvenation of photo- and chronically-aged facial skin has become a really hot topic for aesthetic clinicians and their patients over the past two decades. The introduction of the carbon dioxide $\left(\mathrm{CO}_{2}\right)$ followed by the erbium:YAG (Er:YAG) laser for full-face ablative laser resurfacing (ALR) in severely photoaged patients was a large step forward in the late 1990s from conventional mechanical or chemical methods, ${ }^{1,2}$ and remains till today the gold standard in this class of patient. However, the sequelae with ALR were very unpleasant with unsightly crusting and oozing, prolonged erythema, and the strong possibility of textural or pigmentary changes if patients compliance with wound care was not good. ${ }^{3}$ Patients with less-severe skin aging were not willing to undergo ALR, and patient demand in the very early 2000s led to what was termed 'nonablative skin rejuvenation' (NSR). This involved delivering laser energy to give controlled damage in the dermis under a cooled epidermis to protect against epidermal damage, so that dermal repair would then take place with neocollagenesis and remodelling to give skin rejuvenation. ${ }^{4}$ Systems included pulsed dye lasers, diode lasers operating in the near-infrared and the $\mathrm{Nd}: Y A G$ laser at 1064, but particularly at $1320 \mathrm{~nm}$. Unfortunately, although the theory behind NSR was good, the results in clinical practice were disappointing with patient satisfaction rates hovering around $50 \%$ in the short term, and much lower with long term follow-up. ${ }^{5}$ Various strategies were reported to improve these disappointing results, including combining NSR wavelengths in the one session. ${ }^{6}$ Results were better, but not dramatically so.

The industry then looked at ways to avoid the unwanted effects of ALR but with better efficacy than the NSR, and fractionation of the beam was introduced with the near IR wavelength at $1540 \mathrm{~nm}$, nonablative fractional laser resurfacing (NFLR), also known as fractional photothermolysis. ${ }^{7}$ This approach fractionated the laser beam into myriad microbeams, which delivered columns of coagulation in the dermis under a more-or-less intact epidermis, known as micronecrotic columns (MNCs). The results were better, but again there was not a huge surge in patient satisfaction. It was hypothesized that the major problem was the lack of any real harm to the epidermis, so patients ended up with the 'same old epidermis', even though histology showed good neocollagenesis. So, once again, focus returned to the fractional $\mathrm{CO}_{2}$ laser, which was ablative in its action owing to its high water absorption characteristics. Instead of the MNC, the fractional CO2 laser produced microablative columns (MACs) through the epidermis and down into the dermis, with a border of coagulation, surrounded by large volumes of unharmed tissue. From NFLR, clinicians turned to ablative fractional laser resurfacing (AF LR), ${ }^{8}$ and the results were very good: almost the same efficacy as fully AFR, but with much less in the way of sequelae.

Patients are a fickle group, however, and whereas previously they were resigned to the "no pain, no gain" approach, patients today want all of the gain but with none of the pain, or as little as possible. So once again, focus has returned to achieving nonablative skin rejuvenation, but with the necessity to deliver much more efficacy than the previous NSR approaches. In order to achieve an appropriate degree of neocollagenesis and neoelastinogenesis, but with out so much damage that inflammation and excessive erythema would result, a specific temperature range must be reached in the dermis while keeping the epidermis unharmed. This range is suggested to be around $48^{\circ} \mathrm{C}$ to under $60^{\circ} \mathrm{C} .{ }^{9.10}$ On the other hand, the temperature at the epidermis must not exceed around $47^{\circ} \mathrm{C}$ for any length of time, otherwise epidermal damage will definitely occur with the potential for increased downtime and unwanted side effects, ${ }^{9}$ such as post inflammatory hyperpigmentation especially in darker Asian skin types. The concept of stacking multiple pulses of laser energy with a comparatively low fluence has attracted attention, whereby the effect of each successive pulse resulted in a stepped rise in temperature with skin cooling occurring in between each pulse, and the final result equaled or exceeded the tissue effect if the total fluence had been delivered in a single pulse, with less damage extending beyond the target area only. ${ }^{11,12}$ With an appropriate wavelength, pulse energy and repetition rate, it was postulated that this approach in the 'new' NSR technique could achieve the desired temperature for some collagenesis in the dermis, while leaving the uncooled epidermis unharmed. In fact, mild heating of the epidermis has been shown to be helpful in enhancing the epidermal metabolism, including through the induction of beneficial heat shock proteins HSP90 and HSP70. ${ }^{13}$ Given the water absorption characteristics of the long-pulsed neodymium:YAG (Nd:YAG) at $1064 \mathrm{~nm}$, this pilot study was designed to assess the potential for multishot stacked pulses to generate sufficient dermal temperatures to achieve effective NASR without causing any damage to the epidermis, pain, or unwanted side effects. In addition, the accuracy of the laser device's integral skin temperature sensor was checked in real time against measurements with a laboratory-standard infrared thermographic video camera. 


\section{MATERIALS AND METHODS}

Six informed and consenting female volunteers took part in the study, ages ranging from 24 to $35 \mathrm{yr}$. All 6 subjects were of Korean ethnicity with Fitzpatrick type III skin. After any make-up was cleaned off the face, three points were selected on all subjects for the test sites, namely the bilateral cheeks and the glabella, using anatomical landmarks for guides as shown in Fig. 1.

The laser used was a long-pulsed dual wavelength platform incorporating an alexandrite $755 \mathrm{~nm}$ (Alex) laser and a neodymium:YAG $1064 \mathrm{~nm}$ (Nd:YAG) laser (Clarityll ${ }^{\mathrm{TM}}$, Lutronic Corporation, Goyang, South Korea). The 1064 nm $\mathrm{Nd}$ :YAG wavelength was selected for the present study given the better penetration and absorption characteristics at that wavelength for skin rejuvenation. The parameters used were as follows: $12 \mathrm{~mm} \emptyset$ spot size, $20 \mathrm{~ms}$ pulsewidth, fluence of $13 \mathrm{~J} / \mathrm{cm}^{2}$ and repetition rate of 1 $\mathrm{Hz}$. Three consecutive shots were delivered to each of the 3 test sites in no particular order with the system cryogen cooling set to off.

To monitor epidermal temperature, two methods were used. The laser system touch screen Igraphic user interface, GUI) incorporates a temperature monitor display which is driven in real time via the system microprocessor by an infrared heat sensor built-in to the handpiece (Fig. 2). The temperature monitor consists of a series of green, yellow and red LED indicators which light up when a specific temperature was reached, that can be set up by the user $\left( \pm 1^{\circ} \mathrm{C}\right)$, as follows for the present study: single green, $37^{\circ} \mathrm{C}$; double green, $40^{\circ} \mathrm{C}$; double green plus

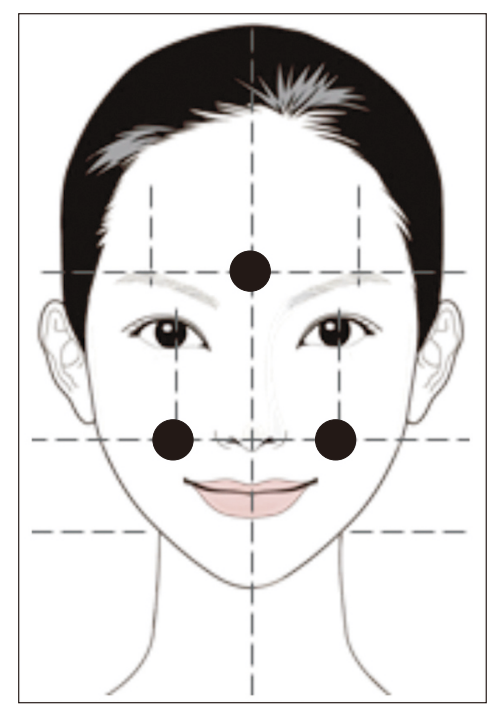

Fig. 1. The three treatment points shown with the anatomical landmarks used to identify them. yellow, $42^{\circ} \mathrm{C}^{\prime}$ double green, double yellow, $46^{\circ} \mathrm{C}$; if the red LED should light up, then the skin temperature has reached or exceeded $47^{\circ} \mathrm{C}$ and the system will automatically stop laser emission to prevent epidermal damage from overheating. For user convenience, the on-screen LED indicators are echoed in similar patterns on LEDs in the smart handpiece. The laser system GUI LED display was photographed to record the LED indicator display after each shot of the three stacked pulses per target area.

In addition to the system built-in monitor, a thermographic video camera was set up to record the skin temperature (FLIR A325, FLIR ${ }^{\circledR}$ Systems, Portland, OR, USA). This system is capable of real-tine temperature measurement at a specific site (or sites) with accuracy up to $\pm 0.001^{\circ} \mathrm{C}$ and data transfer rates to a computer and monitor of $1 \mathrm{~Gb} / \mathrm{s}$, allowing for continuous measurements every millisecond with real-time recording and display. Recording was started a few seconds before the first of the three shots to establish the baseline temperature, and recording was continued for around 20 seconds after each set of stacked pulses to ascertain skin temperature behaviour. All data were stored on a PC for subsequent analysis based on a Microsoft ${ }^{\circledR}$ Excel $^{\top \mathrm{M}}$ database generated by the software which is part of the FLIR camera package. Measurements with the camera were compared for accuracy with those recorded by the laser system integral sensor in the handpiece, as displayed on the GUI LED indicator. In addition to temperature measurement, a thermographic image of the entire field was also recorded to enable matching the recorded data accurately with the

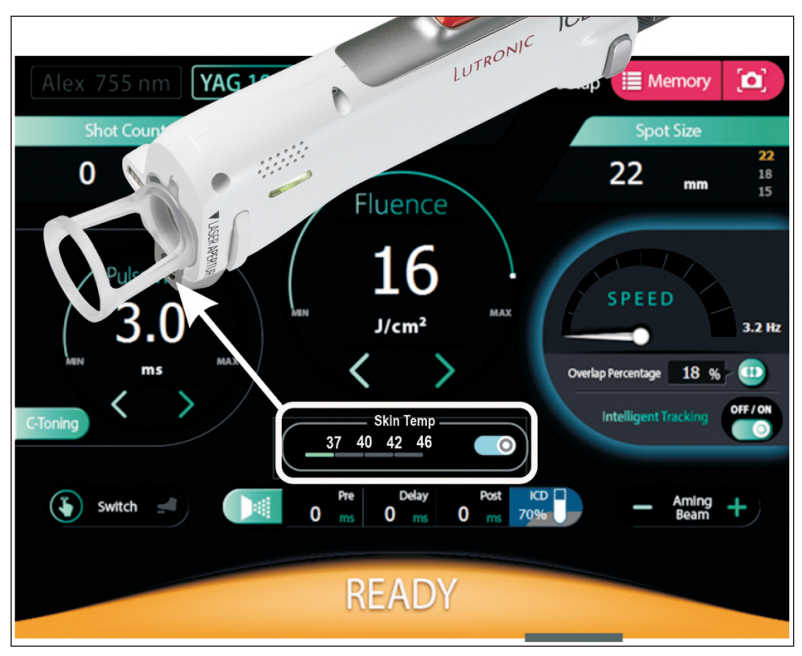

Fig. 2. The GUI (touch screen) of the long-pulsed Nd:YAG laser used in the present study with the skin temperature indicator (white bounding box) and the infrared temperature sensor indicated on the superimposed handpiece (white arrow) (Courtesy, Lutronic Corporation, Goyang, South Korea). 
specific site irradiated.

No anesthesia wa used: subjects assessed discomfort during and after the treatments, and the treatment sites were monitored grossly for up to $2 \mathrm{hr}$ to assess the appearance of any adverse events such as excessive erythema, edema, blistering and so on. Subjects were asked to report any such delayed occurrence over the following $24 \mathrm{hr}$.

\section{RESULTS}

All test shots were successfully delivered on all 6 subjects, and the thermographic records were completed. All subjects described a feeling of mild heat during each multishot with no discomfort during or after the treatments. Mild transient localized erythema was noted at each site, which resolved after an hour or so. No edema was seen, and no other adverse event was observed by the investigator or the subjects after the follow-up period.

The same general temperature pattern was observed for each set of shots for the 3 sites on all 6 patients, whereby a series of increased skin temperatures was recorded in a step-wise manner for each of the single shots in the triple stack, with a decrease in temperature seen between shots on the thermographic data, followed by a steady decrease in skin temperature back towards the baseline value after the third and final multishot. Fig. 3 shows a composite of a representative subject's thermo-

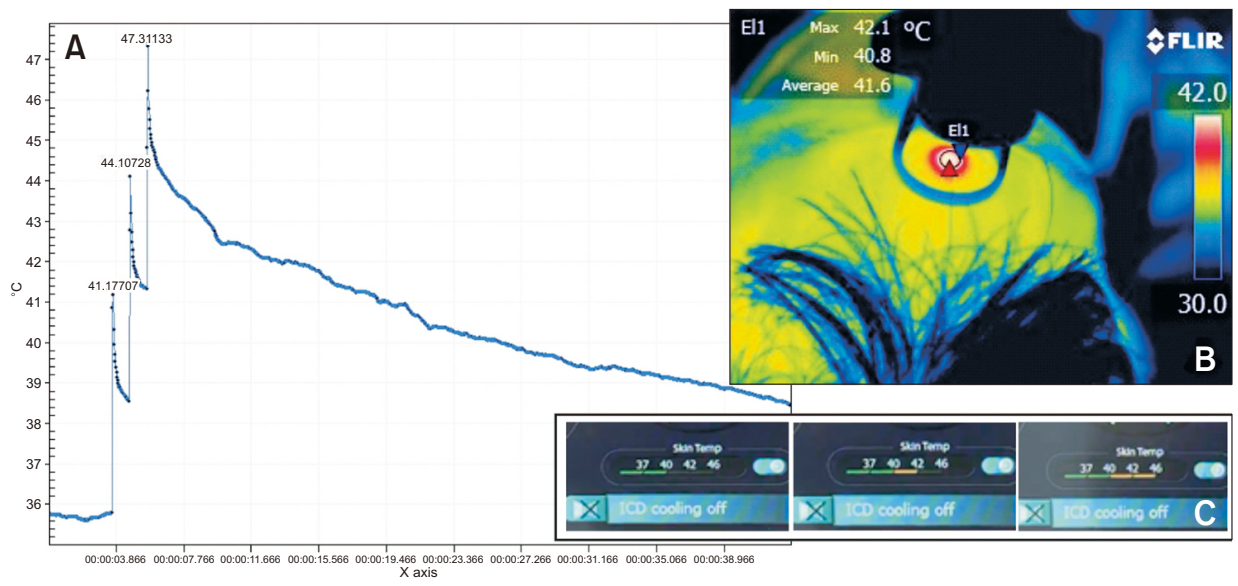

Fig. 3. Example of temperature records for a glabellar site. (A) Temperature gradients graph for each of the three stacked shots and the postshot follow-up, drawn from the Excel raw data. (B) Thermographic image of the face showing the treated site, with the cold handpiece seen in black. (C) Examples of the photos taken of the GUI skin temperature indicators for each shot, with the LEDs illuminated. Please see the text for details.

Table 1. Temperatures reached on each shot of the triple-stacked pulses on the bilateral cheeks and glabella as measured by near-IR video thermography, with the mean temperature \pm standard deviation, and the reading of thesystem LED skin temperature indicator

\begin{tabular}{|c|c|c|c|c|c|c|c|c|c|c|}
\hline \multirow{3}{*}{ No } & \multicolumn{10}{|c|}{ Camera ${ }^{\circ} \mathrm{C} /$ shot } \\
\hline & \multicolumn{4}{|c|}{ Left Cheek } & \multicolumn{3}{|c|}{ Right Cheek } & \multicolumn{3}{|c|}{ Glabella } \\
\hline & $B L$ & 1 & 2 & 3 & 1 & 2 & 3 & 1 & B & C \\
\hline 1 & 35.80 & 39.14 & 41.86 & 44.31 & 38.21 & 40.65 & 43.61 & 39.21 & 42.38 & 44.38 \\
\hline GUI & & G & GG & GGY & G & GG & GGY & G & GG & GGY \\
\hline 2 & 35.35 & 41.24 & 44.72 & 48.22 & 37.51 & 40.02 & 42.79 & 42.75 & 45.1 & 48.77 \\
\hline GUI & & GG & GG & GGY & G & GG & GGY & G & GG & GGYY \\
\hline 3 & 36.05 & 40.39 & 42.07 & 44.48 & 39.32 & 41.42 & 43.92 & 39.81 & 42.29 & 44.36 \\
\hline GUI & & G & $\mathrm{GG}$ & GGY & GG & GG & GGY & $\mathrm{GG}$ & $\mathrm{GG}$ & GGY \\
\hline 4 & 35.81 & 40.89 & 43.46 & 45.25 & 40.99 & 42.96 & 45.53 & 41.18 & 44.11 & 47.21 \\
\hline GUI & & GG & GG & GGY & GG & GG & GGY & $\mathrm{GG}$ & GG & GGYY \\
\hline 5 & 35.31 & 39.20 & 41.82 & 44.24 & 39.32 & 41.97 & 44.68 & 42.88 & 45.65 & 47.07 \\
\hline GUI & & GG & GG & GGY & G & GG & GGY & G & GG & GGYY \\
\hline 6 & 35.65 & 38.09 & 40.34 & 42.67 & 39.16 & 42.04 & 43.65 & 39.18 & 43.71 & 45.32 \\
\hline GUI & & $\mathrm{GG}$ & $\mathrm{GG}$ & GGY & $\mathrm{GG}$ & $\mathrm{GG}$ & GGY & $\mathrm{GG}$ & $\mathrm{GG}$ & GGY \\
\hline Ave & 35.66 & 39.83 & 42.38 & 44.86 & 39.09 & 41.68 & 44.03 & 40.84 & 43.87 & 46.19 \\
\hline SD & 0.26 & 1.10 & 1.39 & 1.69 & 1.52 & 1.06 & 0.87 & 1.55 & 1.25 & 1.63 \\
\hline
\end{tabular}

No, Patient number; BL, baseline temp ${ }^{\circ} \mathrm{C}$; Ave, mean; SD, Standard deviation.

GUI, Temperature sensor on the touch screen: G, green; Y, yellow (For the temperatures corresponding to the LEDs displayed, please see the text). 


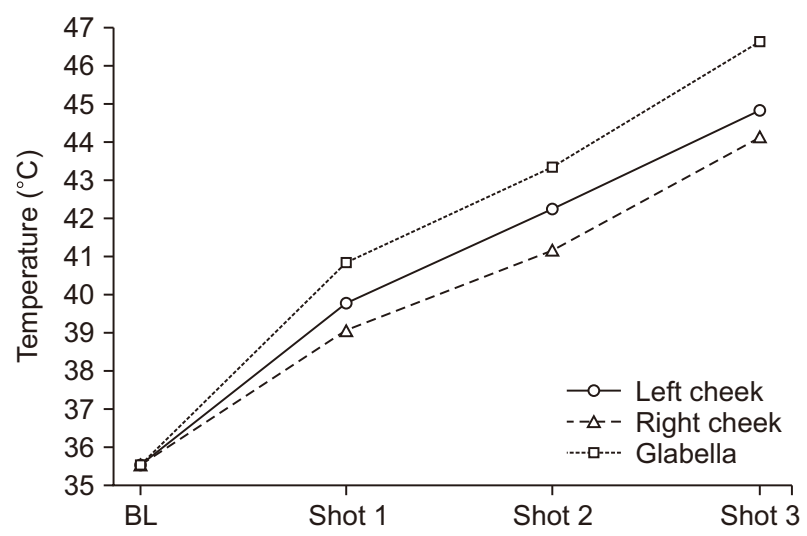

Fig. 4. The mean peak temperatures reached for each shot on both cheeks and glabella.

graphic data illustrating the plotted temperature gradients from a forehead triple stacked shot based on the Excel data (Fig. 3A), an example of a typical thermographic image to identify the target site (Fig. 3B) and examples of the system GUI temperature monitor display for each shot showing two green LEDs $\left(\approx 40^{\circ} \mathrm{C}\right)$, one yellow LED $(\approx$ $\left.42^{\circ} \mathrm{C}\right)$ and two yellow LEDs $\left(\approx 46^{\circ} \mathrm{C}\right)$ corresponding to the skin temperature recorded by the temperature sensor on the handpiece (Fig. 3C). When the temperature readings from the thermographic video camera were compared with those displayed by the LEDs on the GUI, driven from the integral handpiece thermal sensor, the degree of accuracy was ideal for assessing skin temperature measurement during skin rejuvenation in clinical practice, where lower temperatures would be required for mild skin rejuvenation, and higher temperatures for more aggressive rejuvenation, but without ever overheating the epidermis.

Table 1 shows the temperature data for all shots on all six subjects, as well as what the GUI LED indicators showed. The mean temperature at baseline (value \pm SD) was $35.66 \pm 0.26^{\circ} \mathrm{C}$. For shots 1,2 and 3 on the left cheek, the mean temperatures in ${ }^{\circ} \mathrm{C}$ were $39.83 \pm 1.10,42.38 \pm$ 1.39 and $44.86 \pm 1.69$, respectively. For the right cheek they were $39.09 \pm 1.52,41.68 \pm 1.06$, and $44.03 \pm 0.87$, respectively. For the glabella they were $40.84 \pm 1.55,43.87$ \pm 1.25 , and $46.19 \pm 1.63$, respectively. These data are displayed graphically in Fig. 4.

\section{DISCUSSION}

To achieve the current holy grail of nonablative skin rejuvenation (NSR), i.e., maximum gain with minimal pain and no downtime, the temperature in the dermis should be within the range of $48^{\circ} \mathrm{C}$ to under $60^{\circ} \mathrm{C} .10,14 \mathrm{In}$ the low- er portion of this range, production of heat shock protein 47 (HSP47) occurs, ${ }^{15}$ which is a collagen-specific chaperone involved in the formation and stabilization of collagen molecules, helping to ensure the correct formation of the collagen triple helix formation. In the higher range, subcoagulative protein degradation occurs in the collagen bundles whereby the hydrogen bonds holding the fibrils together into fibers are thermally degraded. This leaves the collagen fibers comparatively unharmed, but mildly damaged, and a mild form of the wound healing process is induced which results in mild neocollagenesis and replacement of the degraded hydrogen bonds followed by beneficial reorganization of the extracellular matrix and replenishment of the ground substance. In addition, histological assessment following NSR has demonstrated improvement in the morphology of the Grenz zone, ${ }^{5}$ seen in healthy tissue as a narrow layer of very fine and tightlyknit collagen and elastic fibers, running under and connected to the basement membrane. Improved health of the Grenz zone is echoed by better metabolism in the stratum basale and hence better overall condition of the epidermis.

The problem with the original iteration of NSR in the early 2000s, whereby good dermal reorganization seen histologically was not echoed in a youthful epidermis, lay with the chilling of the epidermis to protect it against thermal damage. As already stated, mild heating of the epidermis is actually beneficial, associated with the production of HSP9O and HSP70 which are chaperones for stratum basale mother keratinocytes function. ${ }^{13}$

During skin rejuvenation using the Genesis or similar techniques with the 1064 LPNDY, up till now the endpoint has been the degree of erythema generated during the treatment. The timing of the appearance of erythema tends to vary from patient to patient, however, and among skin types whereby it can take longer to appear in darker skin types. Real-time infrared thermographic measurement of the temperature rise in the LPNDY-treated skin is the perfect solution, but not everyone has access to a high-accuracy thermographic video camera. The ideal answer is to have an integral and reasonably accurate real-time infrared thermal sensor built in to the handpiece driving some kind of visual temperature display on the system $\mathrm{GUI}$, and that was the case in the system used in the present study. As seen in the Results section, the degree of concordance between the laboratorystandard FLIR camera and the temperatures measured by the integral thermal sensor was ideal for use in clinical rejuvenation practice to deliver specific ranges of heat in the skin for the desired degree of rejuvenation required: 
less heat for mild rejuvenation in mildly-aged skin, and most heat for more moderately-aged skin, but with the safety factor built-in whereby an excessive skin temperature over $47^{\circ} \mathrm{C}$ will instantly sound an audible warning and shut down the system to prevent epidermal thermal damage.

The highest temperatures recorded in the present study occurred in the glabellar site among the three irradiation sites, where the skin overlying the bone is much thinner than in the cheeks, hence the temperatures reached at the glabellar site were slightly higher than at the cheek sites (Table 1, Fig. 4). However, as can be seen from Fig. 3A, which shows the typical temperature gradients for a glabellar site, the peak temperature was held for only milliseconds, with a swift temperature drop off as the microcirculation cooled the treated area down. With temperatures recorded in the epidermis of around $44^{\circ} \mathrm{C}$ to a maximum of $47^{\circ} \mathrm{C}$ with the triple stacked pulses per treatment area, the temperature in the dermis would be within the upper part of the ideal range for NSR $(9,10)$. The present study assed the temperature for three isolated sites. In clinical practice, treatment of larger areas of face would most probably therefore result in mild collagen restructuring with good remodelling, accompanied by beneficial changes in the epidermis. This could arguably achieve the NSR goal of maximum gain in skin rejuvenation of mild to moderately aged skin with no downtime and no pain.

The present study examined the feasibility of using a triple-stacked technique of $\mathrm{Nd}: Y A G$ laser energy at a lower fluence to build up the temperature in the dermis to the ideal range for NSR, while not causing any damage to the uncooled and unanesthetized epidermis. The concept of using multiple stacked shots of laser energy at a lower fluence to achieve safer and better results that the use of a single shot at a higher fluence is well-established. Motta and colleagues demonstrated better skin tightening with fewer side effects using multiple stacked shots of a fractional CO2 laser in a rat model compared with higherfluence single shots. ${ }^{16}$ Lee demonstrated that stacked shots at a low fluence with an Er:YAG laser gave good efficacy with minimal side effects in vaginal rejuvenation. ${ }^{17}$ The present study has shown this concept worked well with the parameters and laser system used, and could offer an ideal minimally-invasive approach for NSR of mildto moderately-aged skin.

\section{CONCLUSIONS}

This pilot in vivo study in human subjects on uncooled and unanesthetized skin showed that a triple stack of lowfluence $1064 \mathrm{~nm} \mathrm{Nd:YAG} \mathrm{laser} \mathrm{shots} \mathrm{on} \mathrm{three} \mathrm{single} \mathrm{tar-}$ get sites, including the thinner skin of the glabella, could achieve an ideal temperature in the dermis for safe and effective nonablative skin rejuvenation for mild- to moderately-aged skin with no unwanted side effects in the epidermis. Further full-face studies performed with the same laser system and parameters, and good follow-up periods, are warranted to bear out the promising results of the present pilot study in clinical NSR practice.

\section{REFERENCES}

1. Trelles MA. Laser ablative resurfacing for photorejuvenation based on more than a decade's experience and 1200 patients: personal observations. J Cosmet Dermatol 2003;2:2-13.

2. Sapijaszko MJ, Zachary CB. Er:YAG laser skin resurfacing. Dermatol Clin 2002;20:87-96.

3. Bernstein LJ, Kauvar AN, Grossman MC, Geronemus RG. The short- and long-term side effects of carbon dioxide laser resurfacing. Dermatol Surg 1997;23:519-25.

4. Zdinak LA, Summerfield ME. Nonablative skin therapies. Ophthalmol Clin North Am 2005;18:237-48, v.

5. Trelles MA, Alvarez X, Martín-Vázquez MJ, Trelles O, Velez M, Levy JL, et al. Assessment of the efficacy of nonablative longpulsed 1064-nm Nd:YAG laser treatment of wrinkles compared at 2, 4, and 6 months. Facial Plast Surg 2005;21:145-53.

6. Trelles MA, Allones I, Levy JL, Calderhead RG, Moreno-Arias GA. Combined nonablative skin rejuvenation with the 595- and 1450-nm lasers. Dermatol Surg 2004;30:1292-8.

7. Hantash BM, Mahmood MB. Fractional photothermolysis: a novel aesthetic laser surgery modality. Dermatol Surg 2007;33:525-34.

8. Geronemus RG. Fractional photothermolysis: current and future applications. Lasers Surg Med 2006;38:169-76.

9. Nelson JS, Majaron B, Kelly KM. What is nonablative photorejuvenation of human skin? Semin Cutan Med Surg 2002;21:23850.

10. Levy AS, Grant RT, Rothaus KO. Radiofrequency physics for minimally invasive aesthetic surgery. Clin Plast Surg 2016:43:551-6.

11. Fitzpatrick RE, Smith SR, Sriprachya-anunt S. Depth of vaporization and the effect of pulse stacking with a high-energy, pulsed carbon dioxide laser. J Am Acad Dermatol 1999;40:61522.

12. Oni G, Robbins D, Bailey S, Brown SA, Kenkel JM. An in vivo histopathological comparison of single and double pulsed modes of a fractionated $\mathrm{CO}_{2}$ laser. Lasers Surg Med 2012;44:410.

13. Berge $U$, Kristensen $P$, Rattan SI. Hormetic modulation of dif- 
ferentiation of normal human epidermal keratinocytes undergoing replicative senescence in vitro. Exp Gerontol 2008;43:65862.

14. Dierickx CC. The role of deep heating for noninvasive skin rejuvenation. Lasers Surg Med 2006;38:799-807.

15. Verrico AK, Moore JV. Expression of the collagen-related heat shock protein HSP47 in fibroblasts treated with hyperthermia or photodynamic therapy. Br J Cancer 1997;76:719-24.

16. Motta MM, Stelini RF, Calderoni DR, Gilioli R, Kharmandayan P. Lower energy and pulse stacking. A safer alternative for skin tightening using fractional $\mathrm{CO}_{2}$ laser. Acta Cir Bras 2016;31:28-
35.

17. Lee MS. Treatment of vaginal relaxation syndrome with an erbium:YAG laser using $90^{\circ}$ and $360^{\circ}$ scanning scopes: a pilot study \& short-term results. Laser Ther 2014;23:129-38.

How to cite this article: Kim YK, Lee HJ, Kim J. Potential efficacy of multiple-shot long-pulsed 1,064-nm Nd:YAG in nonablative skin rejuvenation: a pilot study. Med Lasers 2020; 9:159-165. https://doi.org/10.25289/ML.2020.9.2.159 